\title{
Feature-supervised Compressed Sensing for Microwave Imaging Systems
}

\author{
Chaoqing Tang, Member, IEEE, Guiyun Tian, Senior Member, IEEE, Said Boussakta, Senior Member, IEEE, \\ Jianbo $\mathrm{Wu}$
}

\begin{abstract}
Traditional compressed sensing (CS) applications use sparse information for down-sampling but ignore overall system objectives such as feature extraction. This paper jointly designs sensing and feature extraction process to improve efficiency of microwave imaging systems in time, storage, and feature extraction. A feature-supervised compressed sensing (FsCS) is proposed for cases where not all data contribute to feature extraction. Compared to traditional spatial-spectral sweep and CS solutions, a feature constraint is added in designing CS measurement matrix. More efficient sensing and feature extraction are achieved because only the data contributing to feature extraction is sampled and reconstructed. To improve time efficiency of $\mathrm{CS}$ reconstruction, an aligned spatial-spectral sensing (ASSS) is involved in FsCS to enable joint reconstruction. The proposed scheme is validated in an open-ended waveguide imaging system for low-energy impact damage feature detection. The experimental results demonstrate one order of magnitude improve in time and two orders of improvement in data compression ratio compare to state-of-the-art method while preserving interested feature. This paper can inspire joint sensing-processing designs for more intelligent industrial processes.
\end{abstract}

Index Terms-Compressed sensing, feature extraction, joint optimization, microwave imaging.

\section{INTRODUCTION}

$\mathbf{M}$ ICROWAVE imaging systems use electromagnetic waves in microwave regime to get spatial and spectral response of specimen under test (SUT). It's non-contact, nondestructive and convenient-to-obtain properties make it widely used in nondestructive testing and evaluation and ground penetration radar applications [1]. It's non-ionizing microwave radiation does not cause changes on the atomic and molecular level and is much less harmful to biological tissues compared to ionizing radiation like $\mathrm{X}$-ray, this property makes it suitable for medical applications like breast cancer diagnostics [2]. The spatial and spectral responses of different pixels are implemented by spatial-spectral sweep [3], [4]. Fig. 1 shows the overall diagram of a microwave imaging system. The microwave probe emits microwaves to a pixel on the SUT

Manuscript received $\mathrm{xxx}$; revised $\mathrm{xxx}$; accepted $\mathrm{xxx}$. This work was supported in part by Engineering and Physical Sciences Research Council under Grant EP/J012343/1; National Natural Science Foundation of China under Grant 61527803; China Scholarship Council (no. 201506990030). (Corresponding author: Guiyun Tian)

Chaoqing Tang is with Department of Computer Science, University of Oxford, Oxford OX1 3QD, U.K. (e-mail: bill.tang@cs.ox.ac.uk).

Chaoqing Tang, Guiyun Tian, and Said Boussakta are with School of Engineering, Newcastle University, Newcastle upon Tyne NE1 7RU, U.K. (e-mail: c.tang2@ncl.ac.uk; g.y.tian@ncl.ac.uk; Said.Boussakta@ncl.ac.uk).

Guiyun Tian is also with School of Automation Engineering, University of Electronic Science and Technology of China, Chengdu 611731, China.

Jianbo Wu is with School of Manufacturing Science and Engineering, Sichuan University, Chengdu 610065, China. (e-mail: wujianbo@scu.edu.cn)

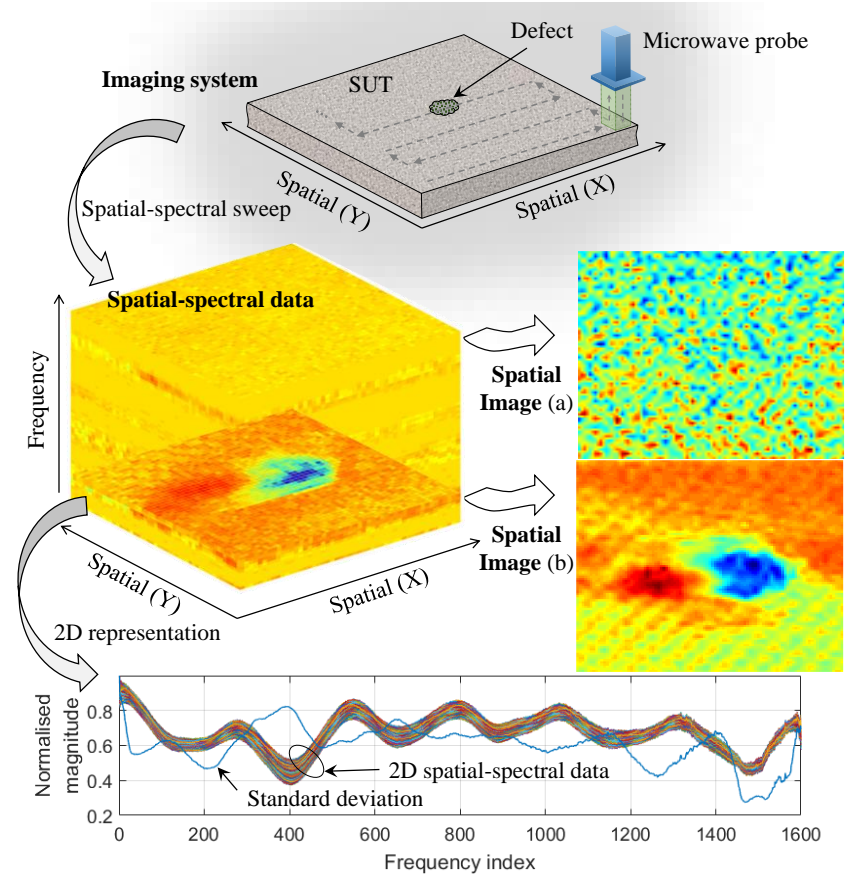

Fig. 1. Diagram for a microwave imaging system.

and waits for reflection signal. Multiple frequency responses are obtained with frequency sweep. Then the probe sweep to the next pixel with spatial sweep. The obtained data can be represented as a 3D cube which contains material property information as shown in Fig. 1. Each horizontal frame is a spatial reflection coefficients image, which can be used for non-destructive testing and evaluation. Each vertical frame for a pixel is the responses at different frequencies, which can be used for material characterization. The spatial stepsize must be small enough to ensure high spatial resolution, but small stepsize leads to drastic increase in the sampling time and data volume. Likewise, more spectral response also increases data volume greatly. This time-consuming issue is crucial for time-sensitive applications [5].

From feature extraction side, the sampled data are usually highly redundant for feature extraction. Due to variation in material property, spatial images for different frequency frames are usually different. Spatial image (b) in Fig. 1 reveals a significant difference in magnitude for some area while image (a) only shows a noisy image. The bottom part of Fig. 1 shows the close-up presentation of the dataset. The multiple frequency reflections of each pixel are plotted as a line vs. frequency index. It is obvious that these lines are highly 
correlated. In fact, only the frequency location where pixel values disperse can show difference within spatial images. Different feature data mining function and indicator may be employed for different systems and problems. For example, the interested frequency locations correspond to large standard deviation value well in this system, so standard deviation is the feature data mining indicator. Mining the feature data is a key step in feature extraction, but it is laborious for big data case or the mining process is complex.

To reduce sampling time, some designs use more hardware such as sensor arrays [6] to parallelly sample multiple spatial pixels. But these designs increase hardware cost. Apart from these hardware solutions, compressed sensing (CS) offers additive benefit from the software side. CS theory first rigidly demonstrated in 2006 [7]. It is fundamentally different from Shanon-Nyquist sampling theorem, which requires far fewer samples than Nyquist sampling rate by solving underdetermined linear equations using sparsity. Sparsity means most values in a signal are zeros, which has wide applicability with proper sparse representation. The down-sampling ability of CS makes it dispense with raster scan in microwave imaging, thus gain more time efficiency. Tang et al. [8] reduce the spatial sampling data in a microwave imaging system based on discrete cosine basis. Yang et al. [9] show that 30\% randomly under-sampled spatial pixels can get good images. Kajbaf et al. [10] also report that $20 \%$ to $30 \%$ spatial pixels of the fullysampled uniform measurements can reconstruct the image in synthetic aperture radar (SAR) systems. 3D SAR systems also reduce time cost in data acquisition [11]. Time-critical systems benefit from CS due to down-sampling. Bevacqua et al. [12] propose a CS-based method for 3D breast cancer microwave imaging, which reduces patient exposure to radiation. The measurement number/data of CS can be reduced to the order of sparse level normally. Besides reducing sampling time, CS also brings other benefits with proper sparse representation. Jung et al. [13] propose using CS in millimeter-wave SAR systems with reduced samples but obtained higher resolution. Stevanović et al. [14] devise a CS strategy to select the optimal orders to consider in the imaging procedure without needing any priori information on the perfect electric conducting target. Gao et al. [15] apply sparse representation in non-destructive defect detection in metals.

The above CS applications have common problems. The reconstruction process is time-consuming for frame-by-frame recovery for the whole spatial-frequency data, although much faster than spatial-spectral sweep. To address these challenges, some papers explore the joint sparsity for situation where multiple signals have the same sparse pattern. Du et al. [16] propose a model to adaptively control sparse level to simultaneously enforce all segments sharing the same active atom set. Wan et al. [17] use multimodal joint sparse representation to improve the performance of biometrics recognition. They represent the received data by a sparse linear combination of potential steering vectors, while constraining the observations from different frequencies subject to sharing the same sparsity pattern. Similar multimodal joint sparse representation also applied in face recognition [18]. Bi et al. [19] investigate a multi-frequency CS model for 2D near-field microwave SAR imaging system. Spatial data of each frequency are represented as a hierarchical tree structure under a wavelet basis and spatial data of different frequencies are modeled as a joint structure. Xia et al. [20] propose a joint kernel sparse representation model, which uses a kernel-induced space with a geodesic exponential kernel for sparse representation. All these methods reduce sensing time and data amount by the down-sampling ability of compressed sensing. However, they bring in large reconstruction burden because they need to reconstruction the same amount of data as that of spatial-spectral sweep method, and there is no benefit for feature extraction.

There are few works on feature extraction-oriented sensing in other fields. Zhang et al. [21] design specific CS for movingtarget imaging by exploiting the geometry information of the defocused results. J. Sun et al. [22] propose a bearing fault detection method directly from compressed sensing data, a similar work is presented in [23]. Du et al. [24] propose CS based impulsive feature detection for wind turbine systems. Chen et al. [25] propose a novel rain streak removal method that is based on an error-optimized sparse representation. For microwave imaging systems, Cetin et al. [26] review the sparse representation in SAR imaging systems, but no literature offers joint sensing and feature extraction design.

In summary, traditional microwave imaging systems have inefficient separate sensing-feature extraction mode. Existed works only apply CS to improve efficiency in sensing and reconstruct the whole dataset, but they ignore the needs of feature extraction, i.e. what and how much sampling data is enough for feature extraction tasks. This paper jointly designs sensing and feature extraction process by a proposed featuresupervised compressed sensing scheme. The main contributions are:

1) A feature constraint is designed for cases where not all data contribute to feature extraction for microwave imaging systems. This feature constraint and sparsity in data defines the data need to be sampled while reserving interested feature, which reduces the latter reconstruction burden.

2) An aligned spatial-spectral sampling (ASSS) scheme is proposed for the sensing under feature constraint. The designed 0/1 Bernoulli measurement matrix is easy for practical implementation. The aligned manner requires less space to store the measurement matrix and laid foundation for joint reconstruction.

3) This paper builds up a joint sparse model for microwave imaging systems. The model enables much faster reconstruction compare to frame-by-frame reconstruction.

4) The application of the proposed method in impact damage detection using open-ended waveguide imaging systems greatly improves efficiency. One order of magnitude improvement in time efficiency and two orders of magnitude improvement in data compression ratio are achieved while preserving the interested feature.

The rest of this paper is organized as follows: Section II presents the proposed schemes. Experiment setup is discussed in section III. Results and discussions are given in section IV before the conclusion in the last section. Table I gives the important notations in this paper. 
TABLE I

NOTATIONS

\begin{tabular}{cc}
\hline \hline Symbol & Description \\
\hline$\lfloor\cdot\rfloor$ & Flooring to get integer \\
$\|\cdot\|_{x}$ & $\ell_{x}$-norm \\
$\|\cdot\|_{x, y}$ & Imposing $\ell_{x}$-norm on columns then $\ell_{y}$-norm on rows to a matrix \\
$\left\{\mathbf{x}_{i}\right\}_{i=1}^{N}$ & Individual term of $\mathbf{x}_{\mathbf{i}}$, where $i=1,2,3, \ldots, N$ \\
{$\left[\mathbf{x}_{i}\right]_{i=1}^{N}$} & Concatenating $\mathbf{x}_{\mathbf{i}}$, where $i=1,2,3, \ldots, N$ \\
\hline \hline
\end{tabular}

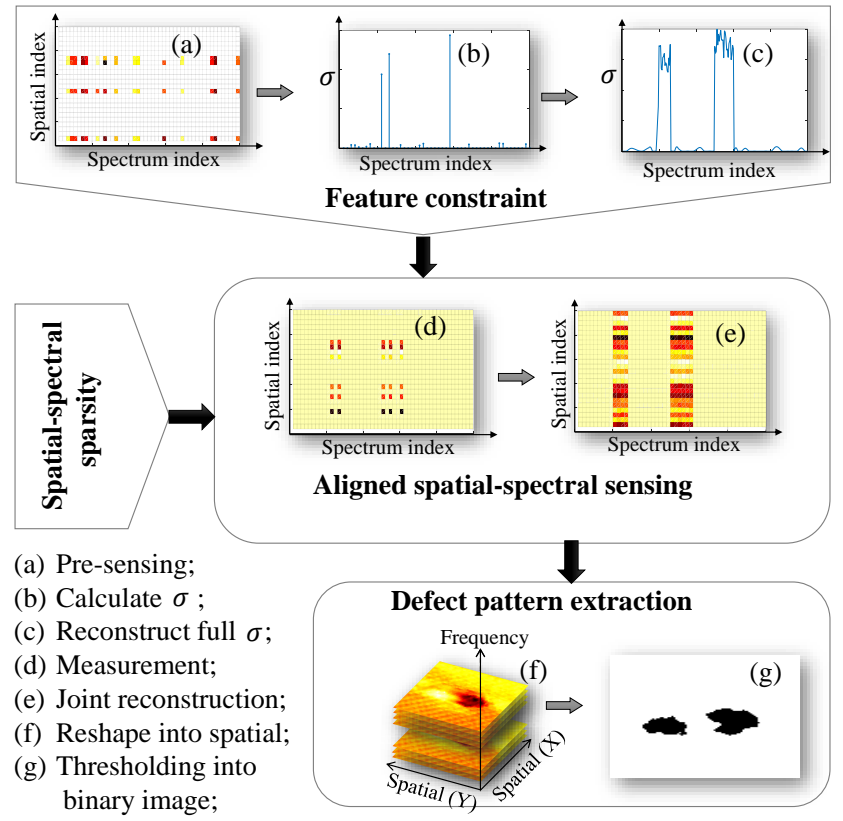

Fig. 2. Diagram of proposed schemes.

\section{THE PROPOSED FEATURE-SUPERVISED COMPRESSED SENSING}

Instead of performing feature extraction in the whole reconstructed spatial-spectral data which involves a laborious reconstruction and feature extraction process as in traditional CS methods, this section proposes feature-supervised CS (FsCS) scheme to reduce the reconstruction burden and innovatively integrate the feature extraction process into the sensing process. The post feature extraction is greatly simplified and reduced data amount. This scheme is applicable to cases where features embedded in small segments of whole data. For time and storage efficiency, only acquiring these data is sufficient for feature extraction. Fig. 2 shows the diagram of the proposed FsCS. FsCS takes the spatial-spectral sparsity and feature constraint as input. The feature constraint blocks integrate part of the feature extraction process to supervise CS. Then aligned spatial-spectral sensing (ASSS) is proposed to sense the spatial-spectral data and speed up the reconstruction. The details are discussed in next subsections.

\section{A. Spatial-spectral sparsity}

This subsection explores the similarity between neighbor frames and frequency response between pixels, aiming to sparsely represent them for the claimed advantages. As shown in Fig. 1, the frequency sweep data of raster scan from microwave imaging system is a 3D signal sampled from frequency/time/spatial domain, denoting as $\Gamma \in \mathbb{R}^{N x \times N y \times n_{f}}$, which can also be regarded as video on the frequency domain. There are $n_{f}$ frames and each frame is a $2 \mathrm{D}$ image with size $N x \times N y$. The sampled data can also be represented as $\boldsymbol{\Gamma}=\left[\boldsymbol{\Gamma}_{i}\right]_{i=1}^{n_{f}} \in \mathbb{R}^{n_{s} \times n_{f}}$, where $n_{s}=N x N y,\left\{\boldsymbol{\Gamma}_{i}\right\}_{i=1}^{n_{f}} \in$ $\mathbb{R}^{n_{s} \times 1}$ is the column-stacked spatial sample of all frames. $\boldsymbol{\Gamma}_{i}$ can be expanded on more compact dictionary like discrete cosine transform (DCT) $\mathbf{D} \in \mathbb{R}^{n_{s} \times n_{s}}$ as $\boldsymbol{\Gamma}_{i}=\mathbf{D S}_{i}$, where $\left\{\mathbf{S}_{i}\right\}_{i=1}^{n_{f}} \in \mathbb{R}^{n_{s} \times 1}$ is the sparse coefficients for each frame. Under such decomposition, neighbor frames usually have same sparse patterns, i.e., they have same non-zero positions but with different values. Denoting the observation with measurement matrix $\boldsymbol{\Phi} \in \mathbb{R}^{m_{s} \times n_{s}}$ for different frequencies with some noise $\mathbf{N}_{i}$ as $\mathbf{Y}_{i}=\mathbf{\Phi} \mathbf{D} \mathbf{S}_{i}+\mathbf{N}_{i}$, the whole observation is

$$
\mathbf{Y}=\mathbf{\Phi D S}+\mathbf{N}=\mathbf{A S}+\mathbf{N}
$$

where $\mathbf{Y}=\left[\mathbf{Y}_{i}\right]_{i=1}^{n_{f}} \in \mathbb{R}^{m_{s} \times n_{f}}, \mathbf{S}=\left[\mathbf{S}_{i}\right]_{i=1}^{n_{f}} \in \mathbb{R}^{n_{s} \times n_{f}}$, $\mathbf{A}=\boldsymbol{\Phi} \mathbf{D}$, and $\mathbf{N}$ is the independently and identically distributed additive measurement noise. Thus, $\mathbf{S}$ has high percentage of zero rows. Then we can seek for the row-sparse matrix $\mathbf{S}$ by solving the following joint optimization problem:

$$
\hat{\mathbf{S}}=\operatorname{argmin} \frac{1}{2} \sum_{i=1}^{n_{f}}\left\|\mathbf{Y}_{i}-\mathbf{A} \mathbf{S}_{i}\right\|_{2}^{2}+\lambda\|\mathbf{S}\|_{2,1}
$$

where $\lambda$ including different subscript variant are Lagrange factors. With the sparse priori information of the unknown $\mathbf{S}$, finding the solution for (2) or the under-determined equations in (1) becomes feasible, because only the non-zero rows and the corresponding column in $\mathbf{A}$ (denote as $\mathbf{A}_{\mathbf{s}}$, also called support in CS theory) contribute to the measurement results $\mathbf{Y}$. If the support is known, (1) becomes well-determined, the non-zero rows of $\mathbf{S}$ can be obtained easily by:

$$
\mathbf{S}_{\mathbf{s}}=\mathbf{A}_{\mathbf{s}}{ }^{-1} \mathbf{Y}
$$

From an implementation point of view, searching for support is using as less weighted column of $\mathbf{A}$ as possible to represent $\mathbf{Y}$. There are many existed methods, which can be categorized into four groups [27]-[30], i.e. the convex optimisation-based methods, greedy algorithms, combinatorial algorithms and Bayesian methods [31]. Although most of the methods are for 1D sparse reconstruction, they can be used to solve the problem in (2) by using only one column of Y to find the support $\mathbf{A}_{\mathbf{s}}$ first, then obtaining the whole solution with (3). Proposing new algorithm to solve (2) is not the key effort of this paper, readers can consult some widely-used method like OMP, CoSaMP, etc. Note that the representation error (the left term in (2)) will be 0 for an optimal solution without noise. However, noise is inevitable in practical applications. The noise performance is related to the noise term in (1) and reconstruction algorithms. For example, the noise bound for successful recovery of the widely-used OMP algorithm is given in [32], another work [33] gives the noise bounds for some common noise like Gaussian noise, Poisson noise, impulse noise etc. These noise bounds are corresponding to 
the residual threshold in reconstruction algorithms. However, in real applications, instead of using complex procedure to estimate the system noise, it is more acceptable to set the residual threshold empirically to balance between reconstruction time and accuracy.

After obtaining $\hat{\mathbf{S}}$, the data can be simply obtained by $\boldsymbol{\Gamma}=$ DS. Likewise, the frequency response of each pixel can be sparsely represented as well. The overall process is the same as the above analysis, the only difference is that substituting $n_{f}$ with $n_{s}$ when only $n_{f}$ shows and switching $n_{f}$ and $n_{s}$ when they show together.

The sparse dictionary can be found empirically or using transforms such as DCT or discrete wavelet transform (DWT) or singular-value decomposition (SVD) on the training data. There are also some blind dictionary learning solutions [34]. The sparsity $K$ can be found by thresholding the sorted absolute cumulate summation of decomposed coefficients on the corresponding sparse dictionary. The threshold can be $95 \%$ empirically, which means the largest $K$ coefficients dominant $95 \%$ of total power.

\section{B. Feature constraint}

Traditional defect feature extraction processes impose feature data mining function (denote as $f(\cdot)$ ) to obtain an indicator for feature data mining. Then the interested feature is extracted from the refined spatial-spectral data. The feature data mining process can be denoted as

$$
\left\{\sigma_{i}\right\}_{i=1}^{n_{f}}=\max \left(f\left(\boldsymbol{\Gamma}_{i}\right)-\delta, 0\right)
$$

where $\delta$ is a threshold, which is set empirically, e.g. set it as the top $20 \%$ of sampled value. $\sigma_{i}$ is the corresponding feature data mining indicator. This process is laborious when $\Gamma$ is in large volume.

Instead of imposing (4) on $\Gamma$ as in traditional CS methods, this paper innovatively uses CS to reconstruct the feature data mining indicator directly. This is based on one fact that $\sigma=$ $\left[\sigma_{i}\right]_{i=1}^{n_{f}}$ is sparse when $\delta$ is large enough. A pre-sensing block is introduced first for this purpose.

$$
\mathbf{y}=\boldsymbol{\Phi}_{f} \sigma
$$

where $\boldsymbol{\Phi}_{f} \in \mathbb{R}^{m_{f} \times n_{f}}$ is the spectral measurement matrix, the detail generation process for this measurement matrix will be introduced in next subsection. $\mathbf{y} \in \mathbb{R}^{m_{f} \times 1}$ is the measurement results. However, $\sigma$ cannot be measured directly. Alternatively, sample value can be used to estimate the population value. In this case, it is using the sampling standard deviation to estimate the population standard deviation by modifying the standard deviation formula from (6a) to (6b) to provide unbiased estimation.

$$
\begin{gathered}
\sigma=\sqrt{\frac{1}{n_{s}} \sum_{i=1}^{n_{s}}\left(x_{i}-\mu\right)^{2}} \\
\sigma_{s}=\sqrt{\frac{1}{m_{s}-1} \sum_{i=1}^{m_{s}}\left(x_{i}-\bar{x}\right)^{2}}
\end{gathered}
$$

where $m_{s}$ is the sample number on each spatial frame. $\mu$ and $\bar{x}$ are the population mean and sample mean respectively. So we can use (7) to substitute (5) by employing (6b) as $f(\cdot)$ and specially design the measurement matrix $\boldsymbol{\Phi}_{\kappa}$,

$$
\text { s.t. }\left\{\begin{array}{l}
\boldsymbol{\Phi}_{\kappa} \in \mathbb{B}^{m_{\kappa} \times n_{\kappa}} \sim \mathrm{B}\left(1, p_{\kappa}\right), \kappa=\left\{s_{1}, f\right\} . \\
\sum \forall \text { column of } \boldsymbol{\Phi}_{\kappa}<2, \quad \sum \forall \text { row of } \boldsymbol{\Phi}_{\kappa}=1 . \\
\mathrm{O}\left(K_{\sigma} \log n_{f}\right) / n_{f} \ll m_{f}=\left\lfloor n_{f} p_{f}\right\rfloor \ll n_{f} .
\end{array}\right.
$$

so that (5) can be rewritten as

$$
\begin{aligned}
\mathbf{y}=\boldsymbol{\Phi}_{f} \sigma & =\boldsymbol{\Phi}_{f} \max (f(\boldsymbol{\Gamma})-\delta, 0) \\
& =\boldsymbol{\Phi}_{f} \max \left(f\left(\boldsymbol{\Phi}_{s 1} \boldsymbol{\Gamma}\right)-\delta, 0\right) \\
& =\max \left(f\left(\boldsymbol{\Phi}_{f}\left(\boldsymbol{\Phi}_{s 1} \boldsymbol{\Gamma}\right)^{T}\right)-\delta, 0\right)
\end{aligned}
$$

where $p_{\kappa}=\left\|\sum \operatorname{column}\left(\Phi_{\kappa}\right)\right\|_{0} / n_{\kappa}$ is sampling percentage, which is the percentage of non-zero column-wise summation to total column number. The binary value in each column decides sampling or not for a signal point. Note that it is only numerically equal to $p_{\kappa}=m_{\kappa} / n_{\kappa}$ under the second constraint for $\Phi_{\kappa}$. If a measurement matrix whose columnwise summation is always non-zeros values (such as deterministic binary block diagonal (DBBD) [35]), the sampling percentage is always $100 \%$, which samples as much data as raster scan. The first two constraints in (7) means that the $p_{\kappa}$ percentage of ' 1 ' in $\boldsymbol{\Phi}_{f}$ and $\boldsymbol{\Phi}_{s 1}$ are located in different row and column, which ensures that $\boldsymbol{\Phi}_{s 1} \boldsymbol{\Gamma}$ is a sample of $\boldsymbol{\Gamma}$ without any scaling. It also ensures $\boldsymbol{\Phi}_{f} f\left(\boldsymbol{\Phi}_{s 1} \boldsymbol{\Gamma}\right)=f\left(\boldsymbol{\Phi}_{f}\left(\boldsymbol{\Phi}_{s 1} \boldsymbol{\Gamma}\right)^{T}\right)$. The third constraint offers successful reconstruction condition on $\sigma . m_{f}$ and $\delta$ can be empirically set in practical applications. The proposed measurement matrices lead to same histogram for sampled data and the whole dataset, so $\delta$ can bet set based on the sampled data. $m_{s 1}$ does not have such constraint because no spatial reconstruction is needed. The reconstruction problem for $\sigma$ becomes

$$
\hat{\sigma}=\operatorname{argmin} \frac{1}{2}\left\|\mathbf{y}-\mathbf{\Phi}_{f} \sigma\right\|_{2}^{2}+\lambda\|\sigma\|_{1}
$$

This is a typical CS reconstruction problem which can be solved using sparse reconstruction algorithm. The data which contains feature $\tilde{\boldsymbol{\Gamma}} \in \mathbb{R}^{n_{s} \times n_{\sigma}} \subset \boldsymbol{\Gamma}$ is the frequency location where $\hat{\sigma}>0$, it also decides the reconstruction burden. $n_{\sigma}$ is number of these frequency locations, which decided by the threshold value $\delta$. Large $\delta$ leads to less reconstruction burden. However, there is no strict limitation on $\delta$. Even if under an extreme case of $\delta=\min (\sigma), \sigma$ can be sparsely represented to meet all the above derivation. So the gain in reconstruction burden is $\rho=n_{\sigma} / n_{f}$ compare to other CS methods. The reconstruction quality of (8) determines the accuracy of feature constraint, which is decided by frequency sampling percentage $p \kappa$ and $m_{s 1}$.

The proposed feature data mining method only samples $p_{s 1}$ subset of spatial pixels and $p_{f}$ subset of their frequency responses and obtains the full feature data extraction indicator value, which will be used to supervise the sensing process.

\section{Aligned spatial-spectral sensing}

Based on the above modeling, a scheme called ASSS is proposed in the following algorithm to down-sample and 
jointly reconstruct the spectral-spatial responses which contains defect information. Besides sparse information, feature constraint is another input for sensing in this paper.

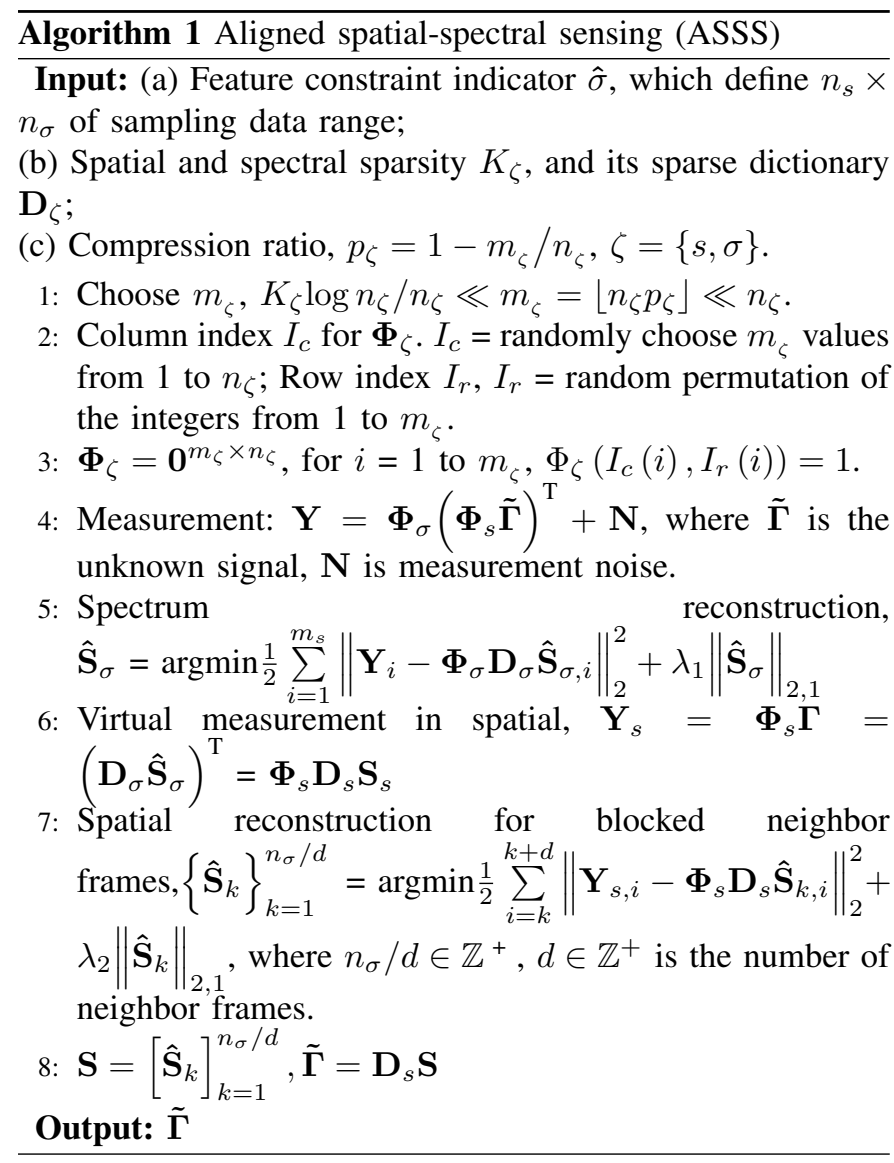

The inputs are the feature constraint indicator obtained in the feature constraint subsection. It defines the specific sensing range which has the feature information and influence the dimension of $\boldsymbol{\Phi}_{\zeta} . K_{\zeta}$ and $\mathbf{D}_{\zeta}$ are obtained in the spatialspectral sparsity section. Compression ratio is the last input which is defined by the user. It is worth noting that $1-n_{\sigma} / n_{f}$ of compression ratio already achieved in the feature constraint process.

Step (1) to (3) use the the results of the spatial-spectral sparsity section and the feature constraint section to design two measurement matrices $\boldsymbol{\Phi}_{\zeta}$ for spatial and spectral sampling. Firstly, $m_{\zeta}$ should be greater than $K_{\zeta}$ for successful reconstruction. The sampling location which denotes as ' 1 ' in the measurement matrix can be implemented by randomly choosing $m_{\zeta}$ values from 1 to $n_{\zeta}$. The sampled locations are randomly distributed to each line of the measurement matrix with the random column and row index. This design ensures that there is no blank data in the measurement results. Each column has one ' 1 ' at most which eliminates duplicate sampling. This design can be used to implement the measurement matrix $\Phi_{f}$ and $\Phi_{s 1}$ in Equ.(7), because it ensures that the sampling results are a subset of original signal without any scaling.

Step (4) implements the measurement model in Equ.(1). After this step, microwave imaging systems get $m_{\sigma} \times m_{s}$ observation in spectral and spatial domain with $\boldsymbol{\Phi}_{\zeta}$. All sampling locations are aligned, i.e., all frames have the same spatial sampling location and all sampled pixels have the same frequency location, as shown in Fig. 2(d). This aligned scheme brings some critical advantages. Firstly, each sampled pixel obtains most frequency sampling points and each sampled frequency point obtains most pixel values, thus ensuring highquality reconstruction for the sampled location and all spatialfrequency data as a result. Secondly, different frames or pixels can share the same measurement matrix, which is easy for hardware and software implementation. Lastly, sharing the same measurement matrix reduces the storage space when saving measurement matrix for reconstruction.

Step (5) and (7) is solving the reconstruction problem in Equ. (2), which can employ methods like OMP or CoSaMP. Step (5) reconstructs the whole frequency response of sampled pixels, these reconstruction results fill the unsampled frames in spatial domain, which works as a virtual measurement process in step (6). All frames have sampled data and these data are in the same location for each frame due to the aligned property. As for the spatial reconstruction in step (7), the $d$ neighbor frames are segmented into the same block which can be jointly reconstructed. When $d=1$, it degrades to frame-by-frame method. Large $d$ brings larger gain in time efficiency, but it needs to consider the degree of dataset that shares same sparse location in same dictionary. In the case study of this paper, $d=10$ makes each block show high joint sparsity. This block-by-block joint reconstruction manner greatly reduces the reconstruction burden. All sparse coefficients of interested frames can be reconstructed in a shifting manner. Step (8) concatenates the all sparse coefficients and get the final image frames.

\section{EXPERIMENTAL SETUP}

FsCS needs to randomly sample in spatial and spectral domain. For random spatial sampling, waveguide imaging systems can use X-Y scanner to locate the probe at arbitrary pixels as shown in Fig. 1. There are also other specially designed hardware for CS making use of spatial masks rather than mechanical scan [36], [37]. For random spectral sampling, the progression in direct digital frequency synthesizer (DDFS) provides fast and reliable arbitrary frequencies output. Modern VNAs use frequency synthesizer gradually. Likewise, there are designs like frequency masks or even spatial-frequency masks [38], [39]. The computationally intensive reconstruction process for large spatial area can go to more powerful computational center like cloud computing, which fits with the IoT structures [40]. Note that the proposed scheme does not specify any sparse reconstruction algorithms as it is not a key research task here. A review of sparse reconstruction algorithms is given in [41].

This paper validates the proposed algorithms in a waveguide imaging system in our lab experiment as shown in Fig. 1 and Fig. 3. A mechanical scanner which carries an open-ended rectangular waveguide probe is used to measure arbitrary point on specimens. The measure location depends on the measurement matrix. A vector network analyzer (Agilent PNA E8363B) is connected to the waveguide probe to emit and 
measure the microwave. Matlab is used to control the network analyzer and mechanical scanner through the GPIB interface, which can cope with both random spatial sampling and random spectral sampling. The reconstruction process is done on a personal computer with Intel i5 4690K CPU and 8GB memory. During the reconstruction process, OMP is used for feature indicator reconstruction, and CoSaMP is used for joint reconstruction, because these greedy algorithms have good trade-off between sampling number, reconstruction accuracy and time. Code for OMP and CoSaMP can be fonud in https://uk.mathworks.com/matlabcentral/fileexchange/32402cosamp-and-omp-for-sparse-recovery. The SUTs are six carbon fibre reinforced polymers (CFRPs) with different impact damage in the center part. The CFRPs are made of Polyphenylene Sulphide (PPS) and have 12 layers of 5H satin balanced carbon fibre woven fabrics. All specimens are in cuboid shape with width $\times$ height $\times$ thickness of $100 \times 130 \times 3.8$ $\mathrm{mm}$. Impact damages are created by a free-fall hammer with mass $2 \mathrm{~kg}$ over the specimen center from different height. The impact point are known in advance with the help of the scale marks on each specimen as shown in Fig. 3. Each specimen is scanned with raster scan and frequency (spatial-spectral) sweep scheme for 20 times with slightly different scan area around the impact point, thus getting enough training data to get the sparsity $K$. The probe scans $99 \times 99$ pixels in each scanning. The frequency band is $18 \mathrm{GHz}$ to $26.5 \mathrm{GHz}$ with 1601 frequency points.

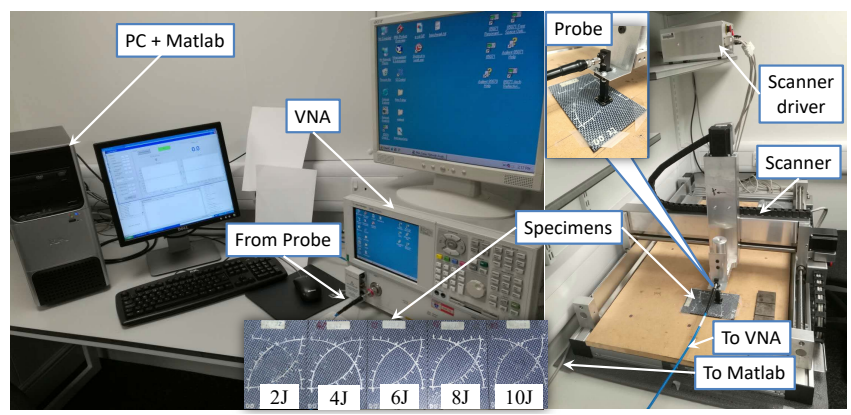

Fig. 3. System setup for the proposed schemes in an open-ended waveguide imaging system for low-energy impact damage detection on CFRP.

\section{RESUlTS AND Discussions}

\section{A. The effectiveness of feature constraint}

The accuracy of feature constraint will directly influence the right spatial-spectral data to sense. We perform spatial-spectral sweep scheme on six different impact energy specimens. Then the proposed scheme is applied to the same specimen and same spatial area.

The probability of detection is used for quantitative validation, it is defined as the probability that the target frequencies are in acceptable error range. In (7), when the threshold $\delta$ is set as the maximum value of $f\left(\tilde{\boldsymbol{\Gamma}}_{i}\right)$, the target frequency is where the maximum value shows. The average probability of detection under such case is shown in Fig. 4 with Monte Carlo method. It is also obvious that the increase in pixel number does not contribute to the detection probability significantly.

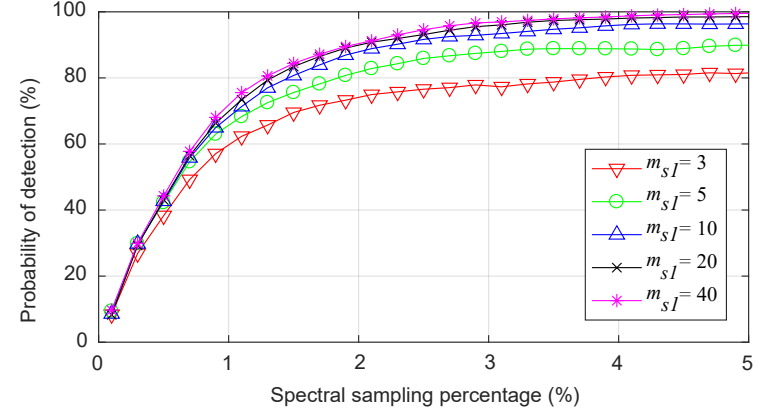

Fig. 4. Average probability of detection of all specimen vs. spectral sampling percentage.

Even just 3 sampling pixels ( $0.03 \%$ of total pixel) get the right target frequency with more than $80 \%$ probability using just $5 \%$ of frequency sampling percentage. 40 pixels $(0.41 \%$ of total pixel, $0.002 \%$ of total data) can find the right target frequency definitely, which demonstrates the powerful detection ability of this algorithm.

\section{B. The performance of aligned spatial-spectral sensing}

The reconstruction quality of ASSS is the source data where defect pattern is extracted. To fully explore the performance of ASSS, all the frequency band is set as interested frequency, i.e. there is no feature extraction constraint applied. In such a case, all spatial-spectral data can be reconstructed for further analysis. The feature constraint only refines the frequency band where ASSS is applied. Fig. 5 presents some results for different spatial and spectral sampling percentage for the $6 \mathrm{~J}$ specimen. The traditional spatial-spectral sweep results are used as references, the state-of-the-art CS method [8][10] and CS method with joint reconstruction [19] are used for comparison from hereunder. With the increase in spatial and frequency sampling percentage, the reconstructed results become more like the reference results. 30\% in spatial and spectral domain already has little visual difference to the reference. The results for CS method that only samples in spatial and CS method use joint reconstruction is used for comparison. These images are visually similar even if there are downsampling in either spatial or spectrum. This means the proposed method obtains similar image as other methods but greatly improves compression ratio.

2D correlation is used to quantitatively compare the reference results and the ASSS results frame-by-frame. 2D correlation evaluates the similarity between the overall shapes of different data sets. The average 2D correlation for all frames in different specimen samples are shown in Fig. 6. Either increase the spatial sampling percentage or spectral sampling percentage improves the reconstruction quality. In this microwave imaging system, only $30 \%$ of spatial and frequency sampling percentage for ASSS obtains $98.2 \%$ similarity as reference results. Even 3\% in both domains gets $69.4 \%$ similarity. The intersecting line between $90 \%$ similarity plane and 2D correlation surface demonstrates the combination case where the similarity is greater than $90 \%$, e.g. $10 \%$ spectral $+20 \%$ 


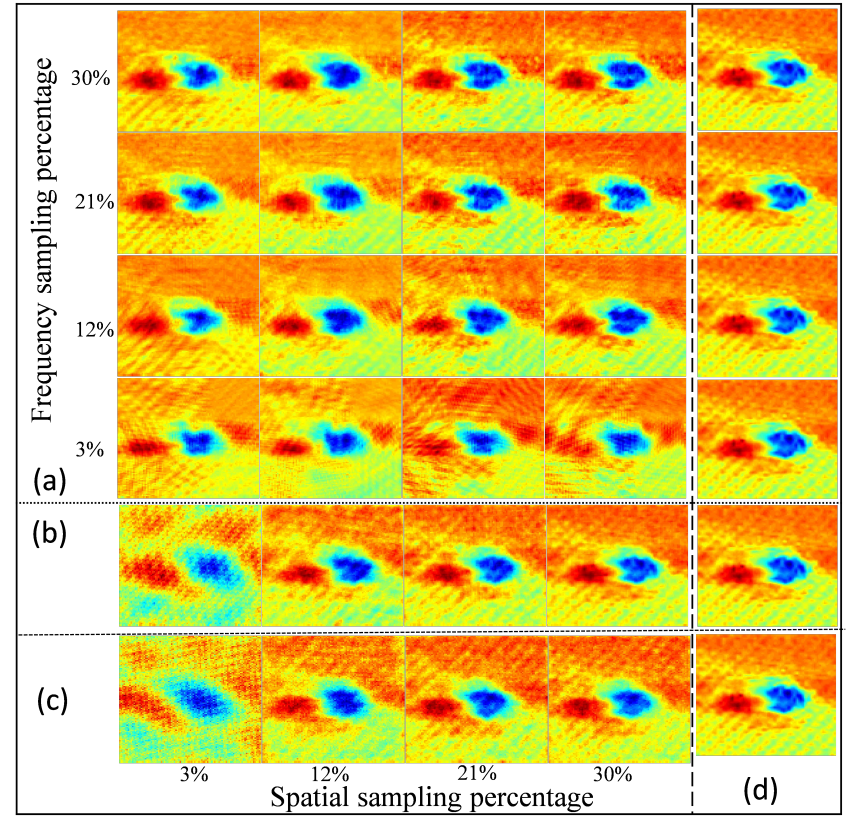

Fig. 5. The images of $6 \mathrm{~J}$ specimen that obtained by (a) the proposed ASSS under various spatial and frequency percentage; and (b) CS; (c) CS with joint reconstruction; and (d) the spatial-spectral sweep as reference.

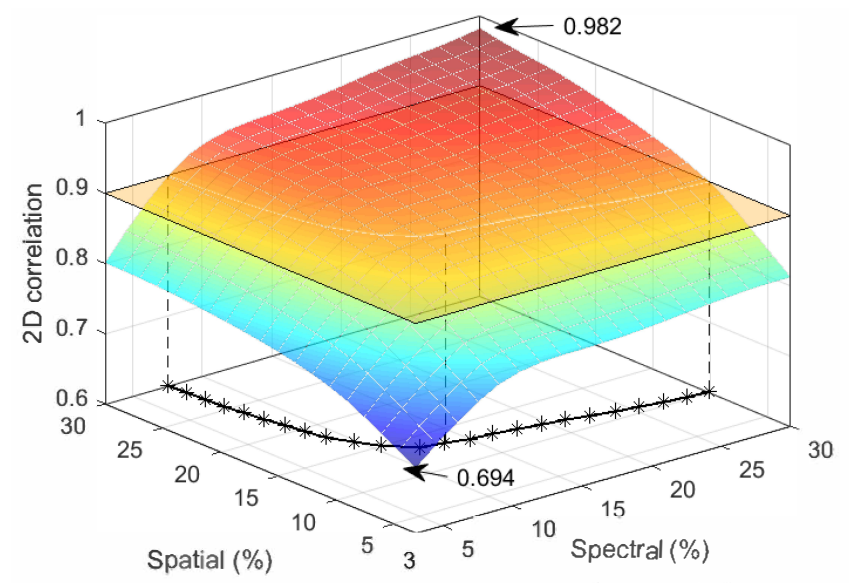

Fig. 6. The average 2D correlation between image of the proposed method and the reference under different spatial and spectral sampling percentage for all specimens.

spatial ( $2 \%$ of whole data) or $15 \%$ spectral $+15 \%$ spatial (2.25\% of whole data).

Quantitative comparison between the images obtained by the proposed method and other methods is shown in Fig. 7. The proposed method with only $15 \%$ of frequency sampling percentage already close to the state-of-the-art method using CS and CS with joint reconstruction. As other methods need to sample the whole frequency band, the proposed method achieves at least $70 \%$ gain in data compression ratio without compromising image quality.

\section{The influence on feature extraction}

The extracted feature is the final output for different processing pipelines. Any information loss or noise influence bringing

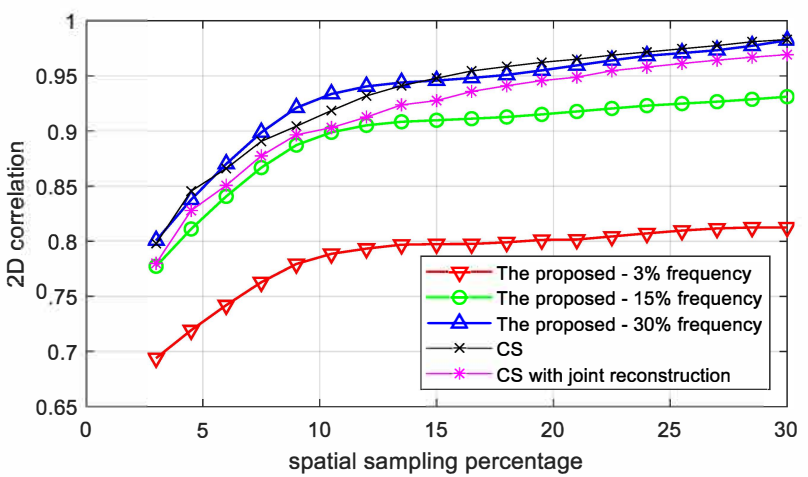

Fig. 7. The average 2D correlation between the images from different methods and the reference for all specimen.

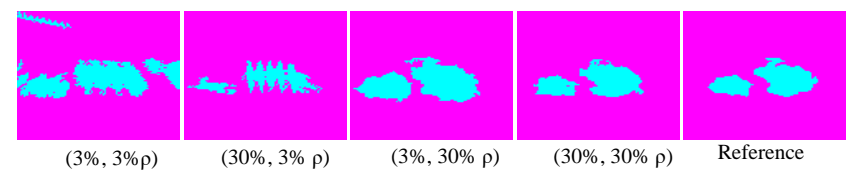

Fig. 8. The extracted defect pattern with various spatial-spectral sampling percentage for the $6 \mathrm{~J}$ specimen.

by the processing pipeline will influence the feature quality eventually. This section presents the influence on feature quality. The size of defect patterns is the interested feature in this study. The defect pattern is extracted from the frequency frames which correspond to large standard deviations, and it is sensed by the proposed scheme by setting the threshold in the feature extraction constraint as $\delta=0.7 \max (\sigma)$. Thus the frequency band to apply ASSS only occupies $\rho=9.4 \%$ of frequency sweep amount. Furthermore, only $p_{\sigma}$ of frequency point are sampled in this interested band. The sampling burden is greatly decreased.

To extract the binary defect pattern, we employ the HTED algorithm from [8] because of its superior de-nosing ability. The HTED algorithm segments the damage region from an image, it is applied to the image that corresponds to the largest standard deviation of the proposed and other methods. Fig. 8 presents some extracted defect pattern for various spatialspectral sampling percentages. More sampling percentage leads to more similar defect pattern as the reference one. Even 3\% of spatial sampling obtains similar defect pattern as reference defect pattern together with $30 \%$ of spectral sampling. Because the reconstructed image under this sampling configuration achieves more than $90 \%$ of correlation with the reference image.

Quantitative evaluation is carried out by the average similarity ratio of defect size between the proposed method and the reference defect pattern by spatial-spectral sweep. Different spatial-spectral sampling percentage, specimens and frequency frames in each specimen are counted in the average. The similarity ratio $(\tau)$ is defined as

$$
\tau=1-\left|S_{\text {rec }}-S_{\text {ref }}\right| / S_{\text {ref }}
$$

where $S_{r e c}$ and $S_{\text {ref }}$ denote reconstructed defect and that of the corresponding reference image. The results are shown in 


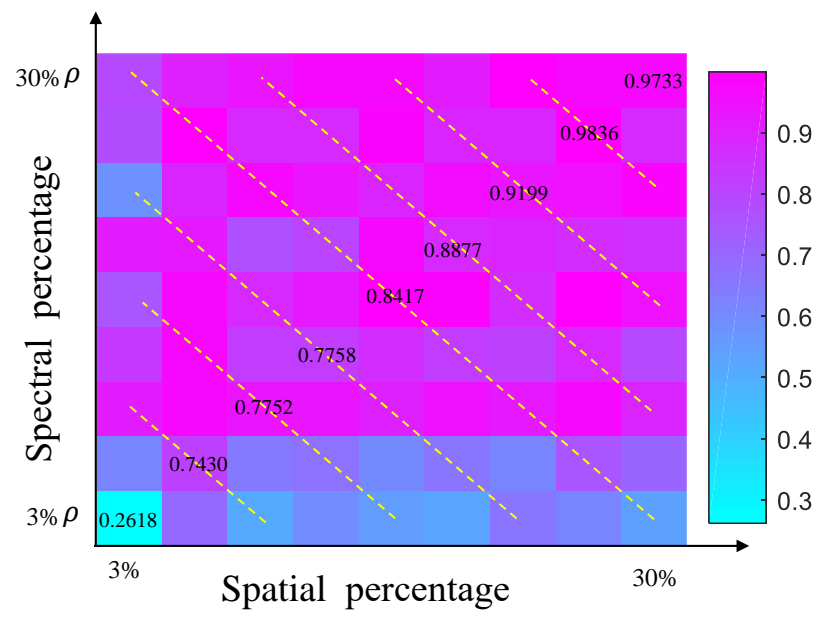

Fig. 9. The average similarity ratio of defect pattern size between the proposed scheme and spatial-spectral sweep results for all specimens.

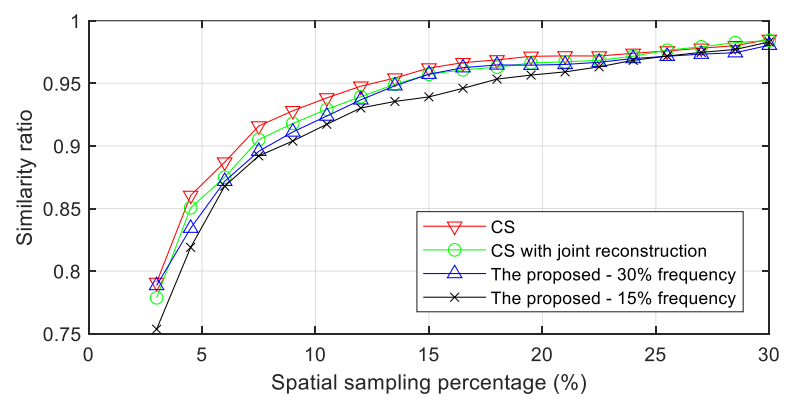

Fig. 10. The average similarity ratio between the defect size obtained by different methods and the reference for all specimen.

Fig. 9. The similarity ratio is very low under $3 \%$ of spatial and spectral sampling rate because the sampling amount is not in the order of $m=O(\alpha \log n)$, which cannot guarantee successful reconstruction. The similarity ratio increases with the increase of spatial and spectral sampling percentage. To highlight this influence, the average similarity of diagonal similarity ratio (The elements covered by the yellow dash line) is calculated and labeled in the figure. It clearly illustrates that more spatial-spectral sampling percentage leads to higher similarity ratio. Fig. 9 also shows that defect patterns extracted from around only $20 \% \rho \times 20 \%=0.37 \%$ of spatial-spectral sweep data already have more than $90 \%$ of similarity ratio as the reference image, which demonstrates FsCS preserves feature integrity even under millesimal level of sampling percentage in this study.

The feature obtained by the proposed method and other methods are shown in Fig. 10, where the spatial-spectral sweep results are used as reference. These results have little difference. The proposed method with only $15 \%$ of frequency sampling percentage already close to the state-of-the-art method, this means $85 \%$ of gain in data compression ratio is achieved than state-of-the-art method.

For more comprehensive comparison, the evaluation of methods in terms of time that obtains image quality larger than 0.95 of $2 \mathrm{D}$ correlation with spatial-spectral sweep, maximum

\begin{tabular}{|c|c|c|c|}
\hline Methods & $\begin{array}{c}\text { Time }>0.952 \mathrm{D} \\
\text { correlation }\end{array}$ & $\begin{array}{c}\text { Max compression } \\
\text { ratio }>0.95 \\
\text { similarity ratio }\end{array}$ & $\begin{array}{l}\text { Reconstruction } \\
\text { burden }\end{array}$ \\
\hline $\begin{array}{l}\text { Spatial-spectral } \\
\text { sweep }\end{array}$ & $186 \mathrm{~min}$ & 0 & Not applicable \\
\hline $\begin{array}{l}\text { Proposed } \\
\text { method }\end{array}$ & $\begin{array}{l}32 \mathrm{~min} \text { sampling; } \\
\text { Rec. }=16 \mathrm{sec}, \\
\approx 1601 \times 0.5 \rho / d\end{array}$ & $\approx 0.21^{2} \rho$ & $\rho$ \\
\hline $\mathrm{CS}$ & $\begin{array}{l}\text { 37min sampling; } \\
\text { Rec. = 782sec, } \\
\approx 1601 \times 0.5\end{array}$ & $\approx 0.21$ & 1 \\
\hline $\begin{array}{l}\text { CS with joint } \\
\text { reconstruction }\end{array}$ & $\begin{array}{c}\text { 37min sampling; } \\
\text { Rec. }=85 \mathrm{sec}, \\
\approx 1601 \times 0.5 / d\end{array}$ & $\approx 0.21$ & 1 \\
\hline
\end{tabular}

Fig. 11. The average performance of different methods in terms of timeconsumption, compression ratio and reconstruction burden.

compression ratio that achieves larger than 0.95 of feature similarity ratio, and reconstruction burden is given in Fig.11 bellow. The time for CS methods consists of both data acquisition time and reconstruction time. The reconstruction time depends on the computation power. Using OMP reconstruction algorithm under $21 \%$ of sampling ratio on Intel Core i54690K CPU in Matlab takes around $0.5 \mathrm{~s}$ for a single frame. This time is same for joint reconstruction. However, joint reconstruction can obtain reconstruction for $d$ frames that share same sparse atom simultaneously. The total reconstruction time can be approximated by multiplying with total frame number. The proposed method has a reconstruction time gain $\rho$ than traditional CS with joint reconstruction. $21 \%$ spatial sampling for traditional CS method obtains more than 0.95 of feature similarity ratio, leading to a compression ratio of 0.21 . The proposed method benefits from the feature constraint and downsampling in spectrum, leading to almost two order of magnitude improvements in compression ratio.

\section{Complexity Analysis}

The computational complexity of the proposed FsCS mainly comes from four parts. 1) Calculate feature indicator for $m_{f}$ sampled frequency frames. 2) Reconstruct $n_{f}$ feature indicator from $m_{f}$ samples. 3) Joint reconstruction for $m_{s}$ frequency frames of length $\rho_{f} n_{f}$ from $m_{f 1}$ samples. 4) Joint reconstruction for $\rho_{f} n_{f}$ spatial frames of length $n_{s}$ from $m_{s}$ samples for every $d$ frames. This paper does not restrict to any sparse reconstruction algorithm. To compare with the CS and CS with joint reconstruction methods, this section assumes they all use OMP (complexity $O(K m n)$ ) for sparse reconstruction. The complexity of calculating feature indicator for a single frame is assumed to be $O(F)$. Joint reconstruction can use the support from single frame iteration for a matrix inverse and a multiplication, which has same complexity as a single frame. So the complexity of the proposed method is $O\left(m_{f} F\right)+O\left(K_{f} m_{f} n_{f}+K_{f} m_{f 1} \rho_{f} n_{f}+\frac{\rho_{f} n_{f}}{d} K_{s} m_{s} n_{s}\right)$. By contrast, OMP method reconstructs all frame in sequence and need to calculate all feature indicator. The complexity is $O\left(K_{s} n_{f} m_{s} n_{s}\right)+O\left(n_{f} F\right)$; CS with 
joint reconstruction jointly reconstructs every $d$ frames and calculates feature indicator for all frames, the complexity is $O\left(K_{s} \frac{n_{f}}{d} m_{s} n_{s}\right)+O\left(n_{f} F\right)$. In practical applications, $m \ll n$ and $n_{f} \ll n_{s}$. So either $O(F)$ or $O\left(K_{s} n_{f} m_{s} n_{s}\right)$ dominants, the proposed method has lower complexity than others.

\section{E. Influence of different category of reconstruction algorithms}

Although reconstruction algorithms are not the key investigation for this paper, different category of methods (i.e. greedy algorithms, convex optimization, combinatorial methods, Bayesian algorithms) may lead to different accuracy and complexity. The above analyses are using greedy algorithms for demonstration for the proposed method and its counterparts. Some general comparison of different category of CS reconstruction methods are given in [27]-[30], a rich resources of code can be found in https://sites.google.com/site/igorcarron2/cs\#reconstruction. As the proposed method and its counterparts are using same CS reconstruction method and the defect region segmentation method, testing different reconstruction algorithms on the same dataset/measurement matrix/sparse basis can show the difference in choosing different category of reconstruction algorithms. So this section chooses popular algorithms from each category to briefly discuss the recovery performance on the above raster scan dataset with the proposed measurement matrix. DCT basis is used for sparse representation again. The chosen algorithms are the widely-used OMP for greedy algorithms, $l_{1}$-magic $\left(l_{1}-\mathrm{m}\right)$ package for convex optimization, chaining pursuit $(\mathrm{CP})$ for combinatorial methods and fast Bayesian matching pursuit (FBMP) [42] for Bayesian methods. Sampling percentage is controlled with the percentage of non-zero columns in the measurement matrix. Same measurement matrix and threshold residual are used for these methods. A quick result is given in Fig.12, where $l_{1}$-magic gives best accuracy and CP gives best time efficiency. FBMP reserves rich detail and the image are more smooth due to noise estimation. To get rid of accidental error, the average $2 \mathrm{D}$ correlation with the raster scan results and the average time consumption per frame are given in Fig. 13 and Fig. 14. The reconstruction accuracy is similar when the sampling percentage is above around $10 \%$ while $\mathrm{CP}$ is the worst. The order of curve before $10 \%$ illustrates the order of requirement on number of measurements reversely. As for complexity, CP is the most efficient method due to its sub-linear complexity. Convex optimization and Bayesian methods are much slower. Greedy algorithm is a good trade-off between them and combinatorial method is also a good choice.

\section{CONCLUSIONS AND FUtURE WORKS}

Traditional CS applications use sparse information for down-sampling but ignore overall system objectives such as feature extraction. This paper presents a joint sensingprocessing design to improve efficiency of microwave imaging systems in time, storage, and feature extraction. A feature constraint is designed for cases where not all data contribute to feature extraction for microwave imaging systems. This feature constraint and sparsity in data defines the data need to

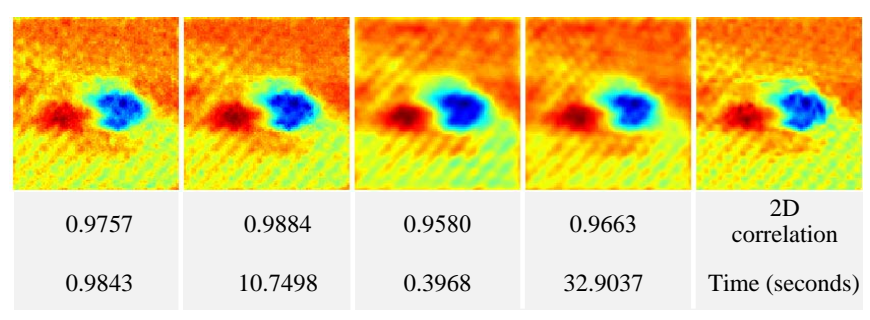

(a)

(b)

(c)

(d)

(e)

Fig. 12. Reconstruction performance for different category of reconstruction algorithms for a frame under $30 \%$ of sampling percentage. (a) Greedy algorithm - OMP; (b) Convex optimization - $l_{1}$-magic; (c) Combinatorial algorithm - Chaining Pursuit; (d) Bayesian algorithm - Fast Bayesian Matching Pursuit; (e) Raster scan image as reference.

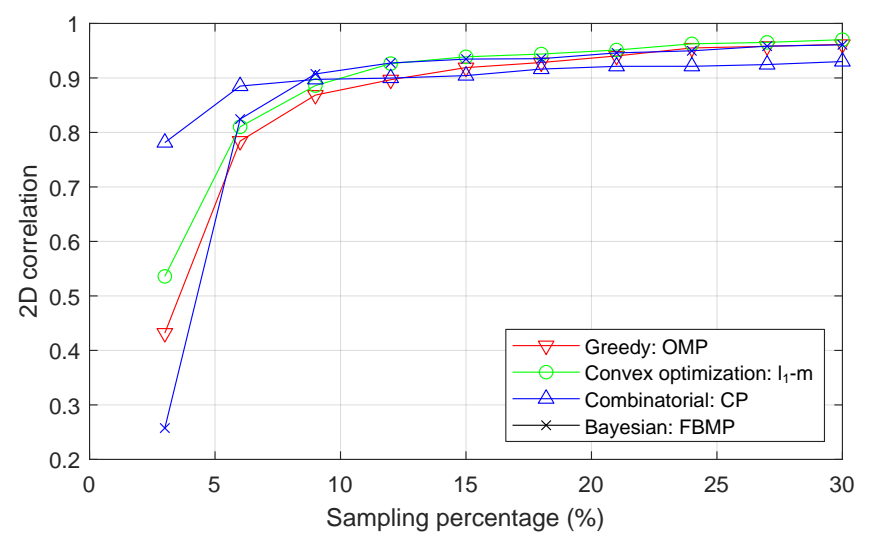

Fig. 13. Average 2D correlation between the CS reconstructed images and raster scan images on the dataset.

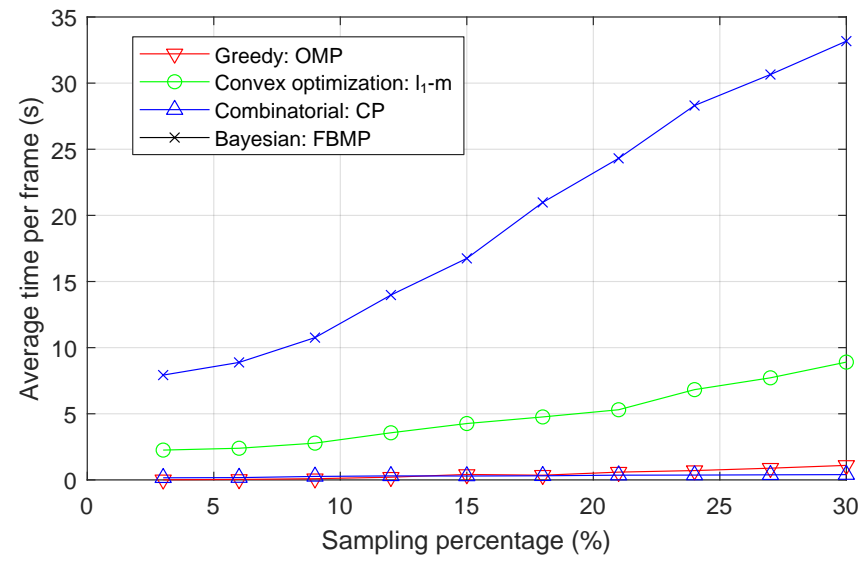

Fig. 14. Average time consumption per frame for different CS reconstruction methods on the dataset.

be sampled while reserving interested feature, which reduces the latter reconstruction burden. An aligned spatial-spectral sampling scheme is proposed for the sensing under feature constraint. The aligned manner requires less space to store the measurement matrix and laid foundation for joint recon- 
struction. Experimental validation in an open-ended waveguide imaging systems for impact damage detection is carried out. One order of magnitude improvement in time efficiency and two orders of magnitude improvements in data compression ratio are achieved while preserving the interested feature.

There are some potential improvements for the proposed method. Firstly, although the DBBD matrix mentioned near equation (7) is useless in the validation system in this paper, it may improve the performance if a measurement system (like program-controlled metasurface [43]) can measure the summation for each row of measurement matrix directly, because DBBD has better incoherence with the DCT basis. Secondly, Kronecker technique [44], [45] divides original signal into segments, due to reduced sensing matrix, more power/computational-efficient sensing can be achieved. The reconstruction quality is equal or even better than global single vector measurement. The reconstruction complexity is also similar as shown in [44], but the problem is that the dimensionality of the CS problem to be solved rapidly grows as the product of the sizes of each dimension as is shown in [46]. So this method is valuable for applications where the sensor side is limited in computation/storage/power. For the scanning system in this manuscript, this will not make much difference, but it is helpful if power-limited microwave sensors are used.

\section{REFERENCES}

[1] C. Li, Z. Peng, T. Y. Huang et al., "A review on recent progress of portable short-range noncontact microwave radar systems," IEEE Trans. Microwave Theory Tech., vol. 65, no. 5, pp. 1692-1706, 2017.

[2] C. P. Hancock, P. Burn, C. I. Duff et al., "A new wave in electrosurgery : A review of existing and introduction to new radio-frequency and microwave therapeutic systems," IEEE Microw. Mag., vol. 16, no. 2, pp. 14-30, 2015.

[3] R. K. Amineh, M. Ravan, A. Trehan et al., "Near-field microwave imaging based on aperture raster scanning with TEM horn antennas," IEEE Trans. Antennas Propag., vol. 59, no. 3, pp. 928-940, 2011.

[4] R. Wang, B.-Z. Wang, Z.-S. Gong et al., "Far-field subwavelength imaging with near-field resonant metalens scanning at microwave frequencies," Sci. Rep., vol. 5, p. 11131, 2015.

[5] R. Chandra, H. Zhou, I. Balasingham et al., "On the opportunities and challenges in microwave medical sensing and imaging," IEEE Trans. on Biomed. Eng., vol. 62, no. 7, pp. 1667-1682, 2015.

[6] S. Jun, Z. Xiaoling, X. Gao et al., "Signal processing for microwave array imaging: TDC and sparse recovery," IEEE Trans. Geosci. Remote Sens., vol. 50, no. 11, pp. 4584-4598, 2012.

[7] E. J. Candès, J. Romberg, and T. Tao, "Robust uncertainty principles: Exact signal reconstruction from highly incomplete frequency information," IEEE Trans. Inf. Theory, vol. 52, no. 2, pp. 489-509, 2006.

[8] C. Tang, G. Y. Tian, K. Li et al., "Smart compressed sensing for online evaluation of CFRP structure integrity," IEEE Trans. Ind. Electron., vol. 64, no. 12, pp. 9608-9617, 2017.

[9] X. Yang, Y. R. Zheng, M. T. Ghasr et al., "Microwave imaging from sparse measurements for near-field synthetic aperture radar," IEEE Trans. Instrum. Meas., vol. 66, no. 10, pp. 2680-2692, 2017.

[10] H. Kajbaf, J. T. Case, Z. Yang et al., "Compressed sensing for sarbased wideband three-dimensional microwave imaging system using non-uniform fast fourier transform," IET Radar Sonar Navig., vol. 7, no. 6, pp. 658-670, 2013.

[11] W. Qiu, J. Zhou, H. Zhao et al., "Three-dimensional sparse turntable microwave imaging based on compressive sensing," IEEE Geosci. Remote Sens. Lett., vol. 12, no. 4, pp. 826-830, 2015.

[12] M. T. Bevacqua and R. Scapaticci, "A compressive sensing approach for $3 \mathrm{~d}$ breast cancer microwave imaging with magnetic nanoparticles as contrast agent," IEEE Trans. Med. Imag., vol. 35, no. 2, pp. 665-673, 2016.
[13] S. H. Jung, Y. S. Cho, R. S. Park et al., "High-resolution millimeterwave ground-based sar imaging via compressed sensing," IEEE Trans. Magn., vol. 54, no. 3, pp. 1-4, 2018.

[14] M. N. Stevanović, L. Crocco, A. R. Djordjević et al., "Higher order sparse microwave imaging of pec scatterers," IEEE Trans. Antennas Propag., vol. 64, no. 3, pp. 988-997, 2016.

[15] B. Gao, W. L. Woo, G. Y. Tian et al., "Unsupervised diagnostic and monitoring of defects using waveguide imaging with adaptive sparse representation," IEEE Trans. Ind. Informat., vol. 12, DOI 10.1109/TII.2015.2492924, no. 1, pp. 405-416, 2016.

[16] Z. Du, X. Chen, H. Zhang et al., "Learning collaborative sparsity structure via nonconvex optimization for feature recognition," IEEE Trans. Ind. Informat., vol. 14, no. 10, pp. 4417-4430, 2018.

[17] L. Wan, G. Han, L. Shu et al., "PD source diagnosis and localization in industrial high-voltage insulation system via multimodal joint sparse representation," IEEE Trans. Ind. Electron., vol. 63, DOI 10.1109/TIE.2016.2520905, no. 4, pp. 2506-2516, 2016.

[18] S. Shekhar, V. M. Patel, N. M. Nasrabadi et al., "Joint sparse representation for robust multimodal biometrics recognition," IEEE Trans. Pattern Anal. Mach. Intell., vol. 36, no. 1, pp. 113-126, 2014.

[19] D. Bi, Y. Xie, L. Ma et al., "Multifrequency compressed sensing for 2$\mathrm{d}$ near-field synthetic aperture radar image reconstruction," IEEE Trans. Instrum. Meas., vol. 66, no. 4, pp. 777-791, 2017.

[20] G. Xia, H. Sun, X. Niu et al., "Keyframe extraction for human motion capture data based on joint kernel sparse representation," IEEE Trans. Ind. Electron., vol. 64, no. 2, pp. 1589-1599, 2017.

[21] X. Zhang, G. Liao, S. Zhu et al., "Efficient compressed sensing method for moving-target imaging by exploiting the geometry information of the defocused results," IEEE Geosci. Remote Sens. Lett., vol. 12, no. 3, pp. 517-521, 2015.

[22] J. Sun, C. Yan, and J. Wen, "Intelligent bearing fault diagnosis method combining compressed data acquisition and deep learning," IEEE Trans. Instrum. Meas., vol. 67, no. 1, pp. 185-195, 2018.

[23] Z. Du, X. Chen, H. Zhang et al., "Feature identification with compressive measurements for machine fault diagnosis," IEEE Trans. Instrum. Meas., vol. 65, DOI 10.1109/TIM.2016.2521223, no. 5, pp. 977-987, 2016.

[24] Z. Du, X. Chen, H. Zhang et al., "Compressed-sensing-based periodic impulsive feature detection for wind turbine systems," IEEE Trans. Ind. Informat., vol. 13, no. 6, pp. 2933-2945, 2017.

[25] B. H. Chen, S. C. Huang, and S. Y. Kuo, "Error-optimized sparse representation for single image rain removal," IEEE Trans. Ind. Electron., vol. 64, no. 8, pp. 6573-6581, 2017.

[26] M. Cetin, I. Stojanovic, O. Onhon et al., "Sparsity-driven synthetic aperture radar imaging: Reconstruction, autofocusing, moving targets, and compressed sensing," IEEE Signal Process. Mag., vol. 31, DOI 10.1109/MSP.2014.2312834, no. 4, pp. 27-40, 2014.

[27] R. E. Carrillo, A. B. Ramirez, G. R. Arce et al., "Robust compressive sensing of sparse signals: a review," EURASIP J. Adv. Signal Process., vol. 2016, DOI 10.1186/s13634-016-0404-5, no. 1, p. 108, 2016. [Online]. Available: https://doi.org/10.1186/s13634-016-0404-5

[28] M. Rani, S. B. Dhok, and R. B. Deshmukh, "A systematic review of compressive sensing: Concepts, implementations and applications," IEEE Access, vol. 6, DOI 10.1109/ACCESS.2018.2793851, pp. 48754894, 2018.

[29] N. Vaswani and J. Zhan, "Recursive recovery of sparse signal sequences from compressive measurements: A review," IEEE Trans. Signal Process., vol. 64, DOI 10.1109/TSP.2016.2539138, no. 13, pp. 3523-3549, 2016.

[30] Z. Zhang, Y. Xu, J. Yang et al., "A survey of sparse representation: algorithms and applications," IEEE Access, vol. 3, pp. 490-530, 2015.

[31] Z. Zhang and B. D. Rao, "Sparse signal recovery with temporally correlated source vectors using sparse bayesian learning," IEEE J. Sel. Topics Signal Process., vol. 5, no. 5, pp. 912-926, 2011.

[32] T. T. Cai and L. Wang, "Orthogonal matching pursuit for sparse signal recovery with noise," IEEE Trans. Inf. Theory, vol. 57, no. 7, pp. 46804688, 2011.

[33] B. Wang, L. Hu, J. An et al., "Recovery error analysis of noisy measurement in compressed sensing," Circ. Syst. Signal Pr., vol. 36, no. 1, pp. 137-155, 2017.

[34] V. Singhal, A. Majumdar, and R. K. Ward, "Semi-supervised deep blind compressed sensing for analysis and reconstruction of biomedical signals from compressive measurements," IEEE Access, vol. 6, DOI 10.1109/ACCESS.2017.2771536, pp. 545-553, 2018.

[35] A. Ravelomanantsoa, H. Rabah, and A. Rouane, "Compressed sensing: A simple deterministic measurement matrix and a fast recovery algorithm," IEEE Trans. Instrum. Meas., vol. 64, DOI 10.1109/TIM.2015.2459471, no. 12, pp. 3405-3413, 2015. 
[36] M. P. Edgar, G. M. Gibson, R. W. Bowman et al., "Simultaneous realtime visible and infrared video with single-pixel detectors," Sci. Rep., vol. 5, p. 10669, 2015.

[37] C. M. Watts, D. Shrekenhamer, J. Montoya et al., "Terahertz compressive imaging with metamaterial spatial light modulators," Nat. Photonics, vol. 8, no. 8, pp. 605-609, 2014.

[38] E. Huang, Q. Ma, and Z. Liu, "Ultrafast imaging using spectral resonance modulation," Sci. Rep., vol. 6, p. 25240, 2016.

[39] J. Hunt, T. Driscoll, A. Mrozack et al., "Metamaterial apertures for computational imaging," Science, vol. 339, no. 6117, pp. 310-3, 2013.

[40] J. Lin, W. Yu, N. Zhang et al., "A survey on internet of things: architecture, enabling technologies, security and privacy, and applications," IEEE Internet Things J., vol. 4, no. 5, pp. 1125-1142, 2017.

[41] E. C. Marques, N. Maciel, L. Naviner et al., "A review of sparse recovery algorithms," IEEE Access, vol. 7, DOI 10.1109/ACCESS.2018.2886471, pp. 1300-1322, 2019.

[42] P. Schniter, L. C. Potter, and J. Ziniel, "Fast bayesian matching pursuit," in 2008 Information Theory and Applications Workshop, DOI 10.1109/ITA.2008.4601068, Conference Proceedings, pp. 326-333, 2008.

[43] Y. B. Li, L. L. Li, B. B. Xu et al., "Transmission-type 2-bit programmable metasurface for single-sensor and single-frequency microwave imaging," Sci. Rep., vol. 6, p. 23731, 2016.

[44] M. F. Duarte and R. G. Baraniuk, "Kronecker compressive sensing," IEEE Trans. Image Process., vol. 21, no. 2, pp. 494-504, 2011.

[45] H. Zanddizari, S. Rajan, and H. Zarrabi, "Increasing the quality of reconstructed signal in compressive sensing utilizing kronecker technique," Biomed. Eng. Lett., vol. 8, no. 2, pp. 239-247, 2018.

[46] G. Coluccia, S. K. Kuiteing, A. Abrardo et al., "Progressive compressed sensing and reconstruction of multidimensional signals using hybrid transform/prediction sparsity model," IEEE Trans. Emerg. Sel. Topics Circuits Syst., vol. 2, no. 3, pp. 340-352, 2012.

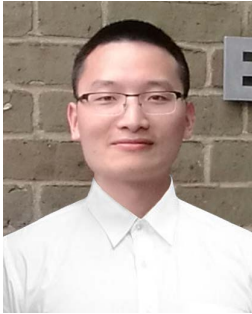

wireless systems.
Chaoqing Tang (M'2019) received the B.Sc. and $\mathrm{Ph} . \mathrm{D}$ degree in electronics engineering from Southwest University, Chongqing, China, in 2015 and Newcastle University, Newcastle upon Tyne, U.K. in 2019, respectively. He is currently a research assistant with the Department of Computer Science in University of Oxford, Oxford, U.K. His research interests are computational methods like compressed sensing, artificial intelligence and optimization theory. The major applications are in non-destructive testing and evaluation, medical image processing and

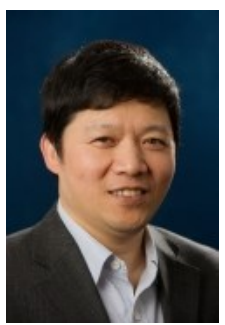

Gui Yun Tian (M'01-SM'03) received his B.Sc. degree and M.Sc. degree from University of Sichuan, Chengdu, China in 1985 and 1988, respectively, and $\mathrm{Ph} . \mathrm{D}$. from University of Derby, Derby, U.K, in 1998. He is currently the Professor of Sensor Technologies at School of Electrical and Electronic Engineering, Newcastle University, U.K. His main interests include electromagnetic sensors, sensor array and sensor network, electromagnetic non-destructive evaluation, advanced signal processing and integrative systems and applications. He has coordinated several research projects from the Engineering and Physical Sciences research Council (EPSRC), Royal Academy of Engineering and FP7. Also he has good collaboration with leading industrial companies such as Airbus, Rolls Royce, BP, nPower, Network Rail and TWI among others.

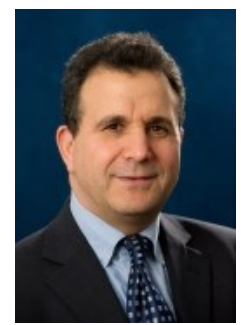

Said Boussakta (S'89-M'90-SM'04) received the Ingenieur d'Etat degree in electronic engineering from the National Polytechnic Institute of Algiers, Algeria, in 1985, and the Ph.D. degree in electrical engineering from Newcastle University, U.K., in 1990 . He is currently a Professor of communications and signal processing with the School of Electrical and Electronic Engineering, Newcastle University. He has authored or co-authored over 200 publications. His research interests include fast DSP algorithms, digital communications, communication network systems, cryptography, and digital signal/image processing. He is a fellow of the IET, and a senior member of the Communications and Signal Processing Societies. He served as the Chair for Signal Processing for Communications Symposium in ICC06, ICC07, ICC08, ICC2010, and ICC2013.

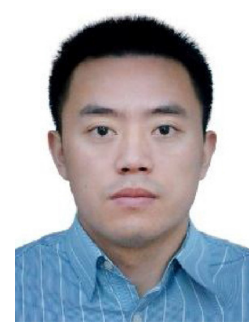

Jianbo Wu was born in Sichuan province, China, in 1986. He received the B.Sc., M.Sc., and Ph.D. degrees from the School of Mechanical Science and Engineering, Huazhong University of Science and Technology, Wuhan, China, in 2009, 2010, and 2014 respectively. $\mathrm{He}$ is currently an associate professor at Sichuan University. His current research interests include magnetic flux leakage testing, eddy current testing, and eddy current pulsed thermography. 